\title{
OPTIMIZING AN ACTUATOR ARRAY FOR THE CONTROL OF MULTI-FREQUENCY NOISE IN AIRCRAFT INTERIORS.
}

\author{
D. L. Palumbo and S. L. Padula \\ NASA Langley Research Center, Hampton, VA 23681
}

\begin{abstract}
Techniques developed for selecting an optimized actuator array for interior noise reduction at a single frequency are extended to the multi-frequency case. Transfer functions for 64 actuators were obtained at 5 frequencies from ground testing the rear section of a fully trimmed DC -9 fuselage. A single loudspeaker facing the left side of the aircraft was the primary source. A combinatorial search procedure (tabu search) was employed to find optimum actuator subsets of from 2 to 16 actuators. Noise reduction predictions derived from the transfer functions were used as a basis for evaluating actuator subsets during optimization.
\end{abstract}

Results indicate that it is necessary to constrain actuator forces during optimization. Unconstrained optimizations selected actuators which require unrealistically large forces. Two methods of constraint are evaluated. It is shown that a fast, but approximate, method yields results equivalent to an accurate, but computationally expensive, method.

\section{Introduction-}

This study was undertaken to better understand the problem of selecting an optimized set of actuators for the control of multi-frequency noise. The ultimate goal is the definition of techniques that can be applied to noise control systems for harmonic noise sources and eventually broadband sources. The immediate application of this technology is to control of the blade passage frequency and multiple harmonics of a propeller aircraft.

The technique builds on past efforts in actuator optimization that have been directed at single frequency systems ${ }^{1,2}$. In brief, transfer functions for the primary and a large set of secondary sources are obtained. Assuming a linear system, the transfer functions are used to estimate noise reduction for different subsets of the secondary sources (noise control actuators). This noise reduction estimate is used as a cost function in a combinatorial search for the optimum subset of

Copyright $\odot$ by the American Institute of Aeronautics and

Astronautics, lnc. No copyright is asserted in the United States under

Title 17, U.S. Code. The U.S. Government has a royaltee-free

license to exercise all rights under the copyright claimed herein for

Government Purposes. All other rights are reserved by the

copyright owner. actuators. A method of tabu search is employed that improves the effectiveness of the search process.

For multi-frequency optimizations, the tabu search algorithm is retained, but with a cost function based on the total noise reduction for 5 frequencies. The cost function is augmented to include control effort penalties which are manipulated to impose control force constraints. Two methods of constraint are evaluated. The uniform constraint method ${ }^{3}$ provides a fast but approximate solution which enables quick optimization trials. These results are compared to those returned using a more exact but computationally expensive solution.

The following sections describe the test facility used to acquire the data, the tabu search algorithm, the constraint methods and the results.

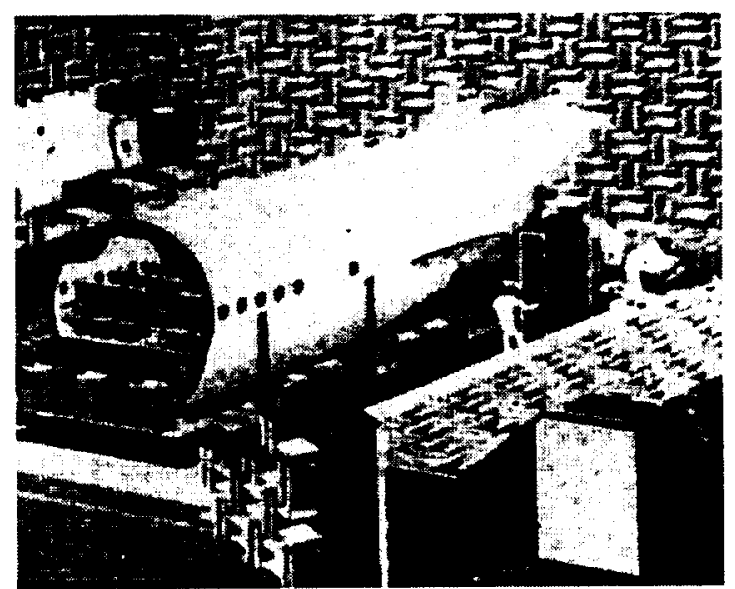

Figure 1. Fuselage Acoustic Research Facility

\section{Euselage Acoustic Research Facility}

Data taken at McDonnell Douglas' Fuselage Acoustic Research Facility (FARF) served as a basis for this study ${ }^{4}$ (see figure 1). The FARF is a large anechoic room which contains the rear section of a DC-9 aircraft minus engines and tail. The interior is complete with seats and trim panels. An isolated volume containing 3 rows of seats was formed by using 2 acoustically treated barriers. The data were originally acquired to support broadband noise control experiments. A large external loudspeaker was used as the primary source. A total of 
18 error microphones were located at head height, one for each seat (15) and 3 in the aisle.

\begin{tabular}{|c|c|c|c|}
\hline 1 & & 46 & 55 \\
\hline 8 & 7 & 36 & 34 \\
\hline & & & \\
\hline & & & \\
\hline 27 & 22 & 47 & 32 \\
\hline 21 & 28 & 56 & 20 \\
\hline 26 & 17 & 40 & 16 \\
\hline & & & \\
\hline 23 & 29 & 30 & 44 \\
\hline 25 & 31 & 41 & 19 \\
\hline WINDOW & WINDOW & WINDOW & WINDOW \\
& & & \\
\hline 48 & 45 & 24 & \\
\hline Front & & & \\
\hline
\end{tabular}

$\leftarrow$ Front of Aircraft

Figure 2. Actuator Location

Actuator Layout. The layout of the piezo-electric actuators is shown in figure 2 for the left side of the aircraft (facing the primary source). The right side of the aircraft had a similar layout for a total of 64 actuators. The actuators were bonded to the interior of the aircraft skin within the bay area formed by the ring frames and longerons.

Broadband transfer functions $(100-1200 \mathrm{~Hz})$ from the primary and secondary sources to the 18 error microphones were obtained. A typical interior pressure spectra due to the primary source is shown in fig. 3. Also shown in figure 3 are five discrete frequencies selected for the simulation $(228 \mathrm{~Hz}, 272 \mathrm{~Hz}, 316 \mathrm{~Hz}$, $360 \mathrm{~Hz}$ and $404 \mathrm{~Hz}$ ). These frequencies were chosen to be equidistant from each other $(44 \mathrm{~Hz})$ and to be in the vicinity of the first 3 modes.

\section{Optimization Overview}

Given a set of $N_{a}$ actuator locations, the immediate goal of an optimization run is to identify a subset of these locations which provides the best performance, i.e. has the potential to reduce the acoustic field to its lowest level. Several combinatorial optimization methods, such as simulated annealing, genetic algorithms and tabu search, are available. Tabu search is used in the present study based on previous experience $^{1,2}$.

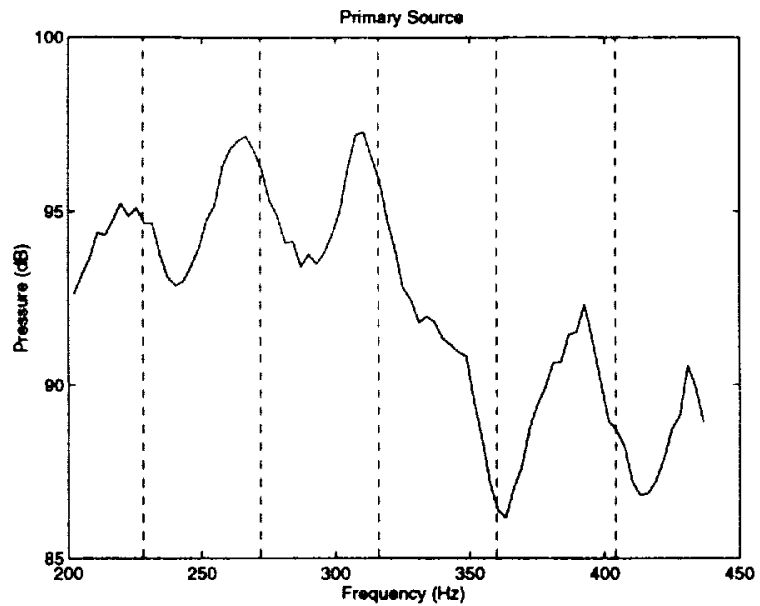

Figure 3. Primary Source Response

To formulate a tabu search problem one defines a state space, a method for moving from state to state, the neighborhood of states which exist around any one state and a cost function. For the actuator selection problem, the set of all possible subsets is the state space. To bound the problem, the subset size, $N_{c}$, is held constant for each search. At any given state, the subset, $N_{c}$, of actuators which represent that state are flagged as "on" while the remainders are flagged as "off". An initial state can be prescribed by the user or generated randomly. A move changes the state by turning one actuator in the subset "off" and another actuator "on". A neighborhood is the set of all states which are one move away from the current state. Finally, the cost function is based on the noise reduction estimate for the subset of actuators which are turned "on".

Each iteration of the tabu search algorithm involves evaluating the cost function for each subset of actuators in the neighborhood of the current state. The move which improves the cost function the most is accepted. If no improving move is identified, then the move which degrades the cost function the least is accepted. The algorithm continues for a predetermined number of iterations. Cycling is avoided by maintaining a list (called the tabu list) of all previous moves. The algorithm is prohibited from reversing any move on the tabu list unless the cost function of that state is superior to any state encountered in previous iterations. The algorithm terminates after reporting the best state encountered during the optimization.

Note that each iteration of tabu search requires $N_{c}^{*}\left(N_{a}\right.$ $N_{c}$ ) evaluations of the cost function. A 14 out of 64 search would then make $14^{*} 50=700$ evaluations per iteration. Typical searches require 25 iterations, 17,500 evaluations. This number of evaluations is small compared to the total number of possible actuator combinations $\left(5 \times 10^{13}\right)$, but can be significant if the cost function requires a lot of computation. It is desirable to choose the least computationally expensive cost 
function that maintains the relative ranking of the actuator sets in the search space. Absolute accuracy is not required.

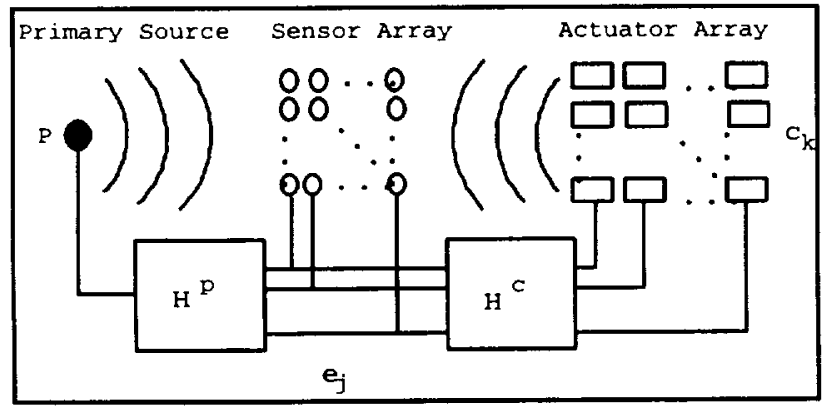

Figure 4. Generic Noise Control Diagram

\section{Optimization Cost Function}

Figure 4 is a block diagram of a generic noise control problem. It is desired to reduce the acoustic noise field, $e$, which is produced by the primary source, $p$, by applying control through the actuator array, $c$. The coupling of the primary and control sources with the acoustic field is given by their respective transfer functions, $\boldsymbol{H}^{p}$ and $\boldsymbol{H}^{\boldsymbol{c}}$. The relationship of these components is written as

$$
e=H^{c} c+H^{p} p
$$

where $c$ is a vector of length $N_{c}$ (the number of actuators in the control array). A measure of the total sound field of a particular control solution, $J$, is the sum of the squared pressures over the $N_{r}$ points in the sensor array which define the response field.

$$
J=e^{*} e,
$$

where ${ }^{*}$ is the complex conjugate transpose. The control is most effective (the performance is optimized) when $J$ is minimized. The control forces which minimize equation (2) are determined by solving a complex least-squares problem. Control algorithms use adaptive procedures such as Filtered-X LMS ${ }^{5}$ to find minimizing control forces. An analytical solution ${ }^{6,7}$ may be expressed as

$$
c=-\left(H^{c^{*}} H^{c}\right)^{-1} H^{c^{*} P}
$$

where $P$ is the pressure field of the primary source, $H^{p} p, H^{c^{*}}$ is the conjugate transpose of the actuator transfer function and $c$ is the vector of control inputs which minimize $J$ in eq. (2).
The optimization procedure uses the average noise reduction as a cost function during the search through the actuator space.

$$
\cos t=10 \log \left(\frac{e^{*} e}{P^{*} P}\right)
$$

This definition of the cost function is inexpensive to evaluate when $N_{c}$ is small and has a unique solution for every subset of actuators considered by the tabu search. The cost function is easily generalized to the multifrequency case by finding the optimum solution for each frequency and then finding the total average noise reduction.

\section{Constraining The Solution}

The expression given by (4) will produce a solution without regard to limitations which may exist on the force that the piezo-electric actuator is able to apply. The actuator force may be limited by many factors, some of which are the design of the actuator, the way it is mounted, and the actuator power supply. Recall that the optimization process uses the control solution to compute the associated noise reduction. To obtain a realizable solution from the optimization process, the control solution must be bounded, or constrained. The control solution is in terms of actuator voltage. To constrain the actuator force, an upper bound is placed on the voltage that the associated control signal may take.

Control effort weighting, or actuator weighting, has been used to limit actuator forces in Filtered-X LMS noise control systems ${ }^{4,8}$. These efforts have been concerned with increasing the robustness of a control system by penalizing the high actuator forces which may result from the response at resonance or instabilities introduced by an ill-conditioned system. To implement the penalty, a term dependent on actuator force is added to the controlled sound field formula, eq. (2).

$$
J=e^{*} e+c^{*} R c
$$

In (5), $R$ is a matrix of actuator weights which determines which actuators are penalized and to what extent. Carrying the $\boldsymbol{R}$ matrix through the minimization solution produces the following equation for actuator forces ${ }^{4}$.

$$
c^{\prime}=-\left(H^{c^{*}} H^{c}+R\right)^{-1} H^{c^{*} P}
$$

It may not be appropriate to uniformly penalize actuator effort in an optimization cost function. To be most accurate, the analytical solution should model the 


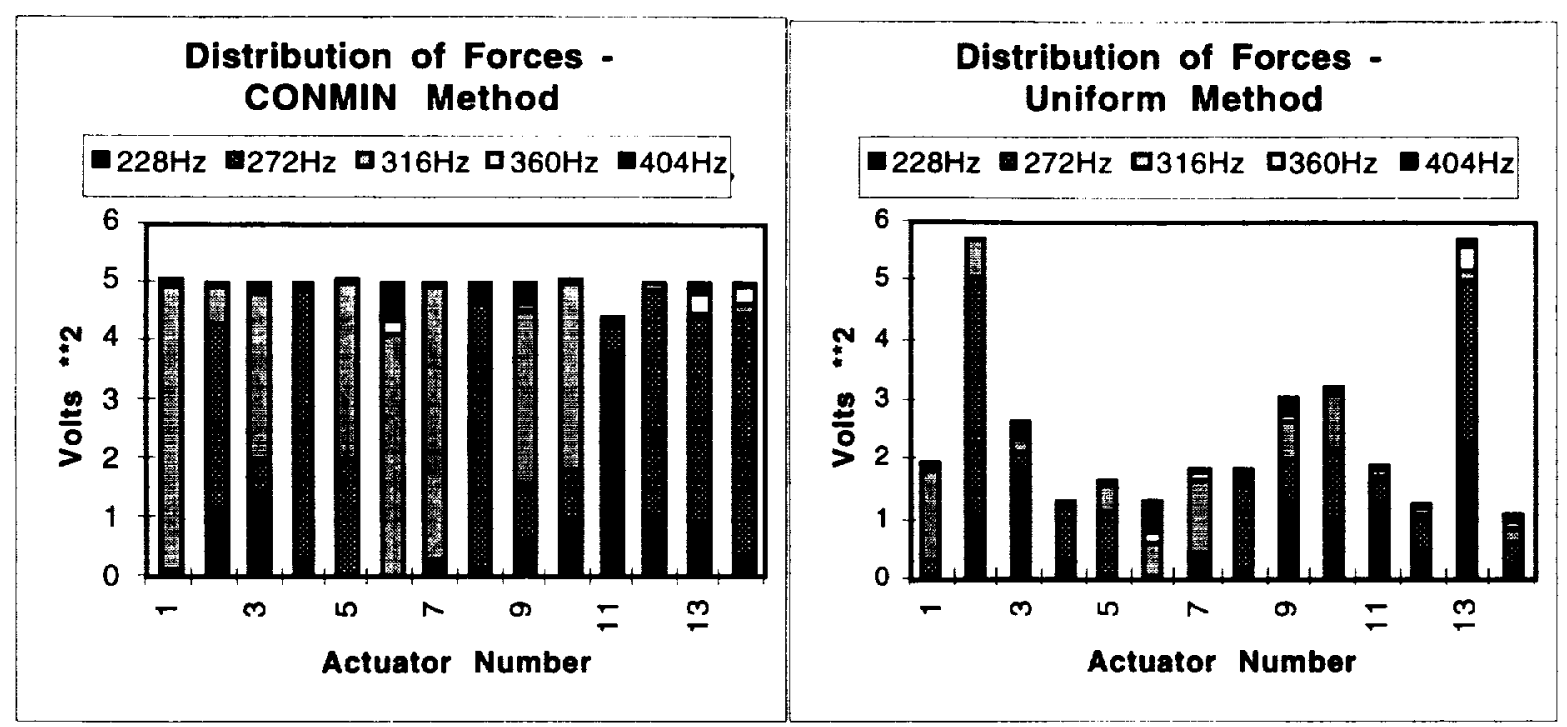

Figure 5. Distribution of Actuator Forces for CONMIN and Uniform Methods

action of the control system faithfully, i.e., each actuator should be allowed to reach its maximum output level such that the greatest noise reduction is achieved. The control weighting matrix, $R$, can be used to limit actuator forces to a prescribed value in the closed form solution (eq. 6) and thus act as a constraint on the solution. However, the correct value of $\boldsymbol{R}$ is not easily derived. To further complicate the matter, the penalty must be distributed over a discrete set of frequencies.

Two methods of forming the control weighting matrix were evaluated: uniform, based on a method described by Rossetti ${ }^{4}$, and CONMIN, a constrained minimization procedure?.

\section{Implementing Constraints}

In equation (6), the $N_{r} \times N_{c}$ transfer matrix $H^{c}$ is formed from a $N_{r} \mathrm{x} N_{a}$ transfer matrix $\boldsymbol{H}^{a}$ by selecting columns associated with the actuators flagged as "on" by tabu search. The calculated values of the control forces, $c$, depend on $\boldsymbol{H}^{c}$ but also depend on the weighting matrix $\boldsymbol{R}$. Note that if $\boldsymbol{R}$ is identically zero or is composed of elements which are small compared to those of $H^{c^{*}} H^{c}$, then the calculated forces, $c$, will minimize noise. On the other hand, if the elements of $R$ dominate (6) then the force solution will reduce control effort. Finding an $\boldsymbol{R}$ which minimizes noise subject to a force constraint is also an optimization problem:

Minimize: $J^{\prime}=e^{*} e+c^{*} R c$

Subject to: $\left\|c_{k}\right\| \leq c_{\max }$ for $k=1,2, \ldots N_{c}$

Design variables : $5^{*} N_{c}$ diagonal elements of $R$

where $\left\|c_{k}\right\|$ is the norm of the control forces for the $k^{\text {th }}$ actuator over the five frequencies. Optimization problem (7) requires that $\left\|c_{k}\right\|$ be smaller than some specified voltage, $c_{\max }$. The CONMIN method solves optimization problem (7) directly. It is a nonlinear programming problem with $5^{*} N_{c}$ design variables and $N_{c}$ constraints.

In the uniform method, an $\boldsymbol{R}$ is found for each frequency by defining a scalar, $r$, such that, $R=r I$ and

$$
r=\sigma_{\max } \frac{\sqrt{P^{*} P}}{\sqrt{c^{*} c}}-\sigma_{\min }^{2}
$$

where $\sigma_{\min }$ and $\sigma_{\max }$ are the minimum and maximum singular values of $\boldsymbol{H}^{a}$. The values of $r$ are calculated once before the tabu search begins. A conservative estimate for $r$ can be found by substituting $c_{\text {max }}$ for $\sqrt{c^{*} c}$ in (8). Better values for $r$, i.e., ones that return more noise reduction while meeting the constraint, can found by trial and error.

Figure 5. illustrates the difference between the CONMIN and uniform methods. The figures plot the mean square voltage, $\left\|c_{k}\right\|^{2}$, for each of 14 actuators at the five frequencies. The CONMIN method raises nearly every actuator to the target voltage, $5.0 \mathrm{~V}^{2}=$ $2.25 \mathrm{Vrms}$, to maximize noise reduction. The force distribution for the uniform method is more random with 2 actuators exceeding the limit. This is not surprising since the uniform method is an inexpensive but approximate solution to the optimization problem. 


\section{Choosing a constraint level.}

A practical constraint level can be estimated by considering actuator power supply specifications. A control algorithm should limit the control signal so that the actuator rms voltage is within power supply specifications. A representative constraint needed to match these operating conditions during simulation can be derived from the system parameters that were used during acquisition of the transfer functions. The transfer functions were taken with a broadband drive signal of $1.5 \mathrm{Vrms}$. This resulted in $80 \mathrm{Vrms}$ at the actuators. If it is assumed that the actuator power supply is rated to operate at $120 \mathrm{Vrms}$, the actuator drive signal could be as high as $2.25 \mathrm{Vrms}$ and stay with power supply specifications. From this point forward all voltages and associated forces are referring to the actuator drive signal, i.e., before the power supply.

Two cases are considered in the following sections, constrained and unconstrained. The unconstrained case uses eq. (3) to compute control signals, $c$. The constrained case uses eq. (6) for $c^{\prime}$, with $R$ determined by either the uniform or CONMIN method subject to $c_{\max }=2.25 \mathrm{Vrms}$.

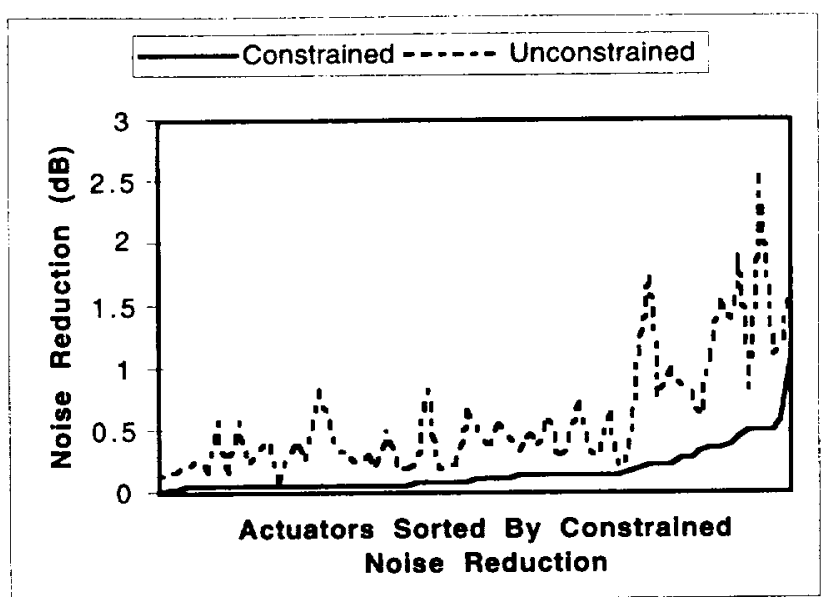

Figure 6. Single Actuator Performance

\section{Effect of Constraints}

Constraints have a pronounced effect on relative actuator performance. Figure 6 shows a plot of noise reduction obtained from individual actuators for both the constrained and unconstrained conditions. The actuators are sorted by the noise reduction they àchieved for the constrained case. It is apparent from the graph that the actuators do not uniformly benefit from reduced constraint levels. Many of the actuators with the

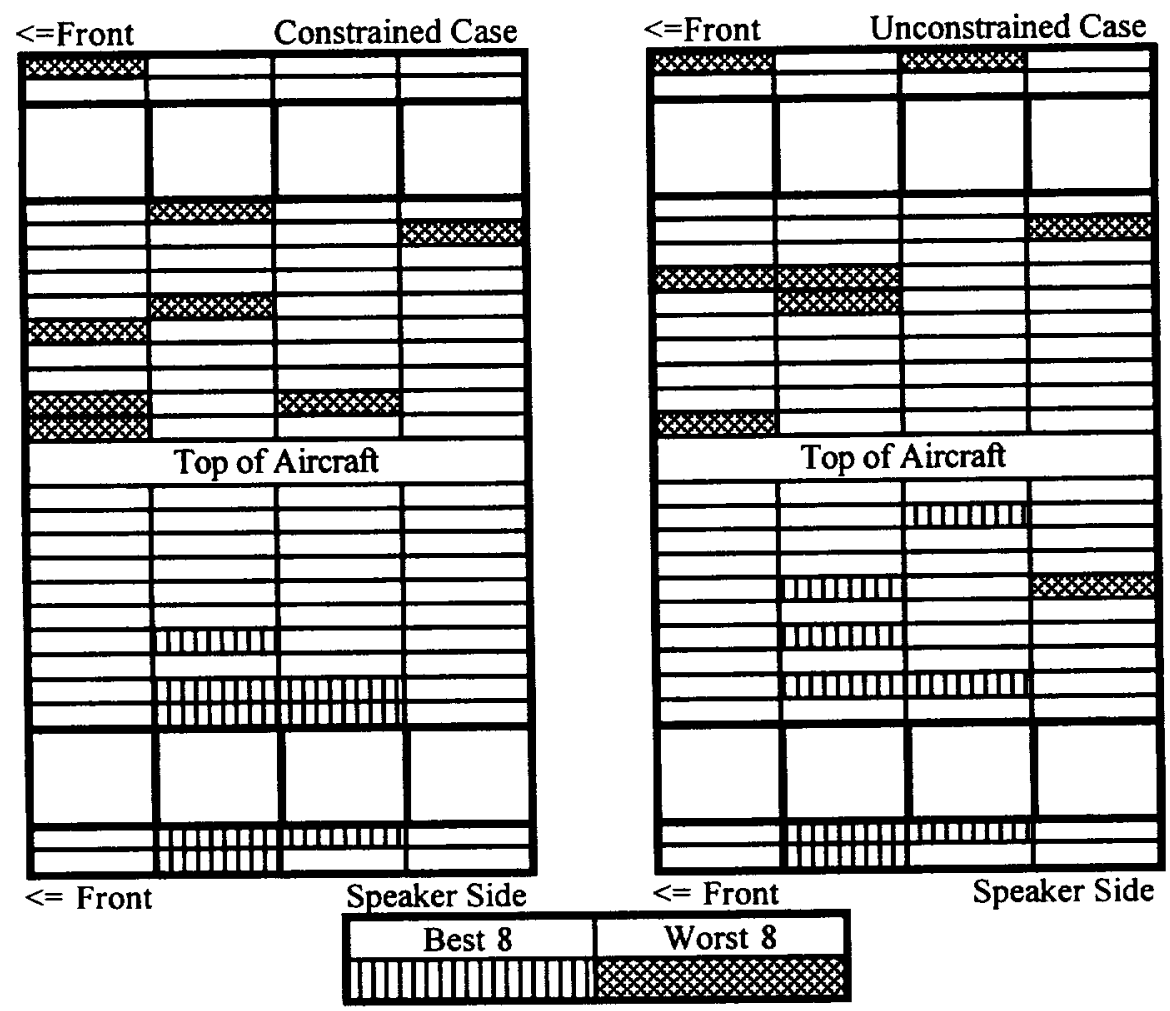

Figure 7. Best and Worst Single Actuators 
poorest constrained noise reduction perform relatively well when the constraints are lifted. This demonstrates the importance of setting a proper constraint level during actuator selection optimization.
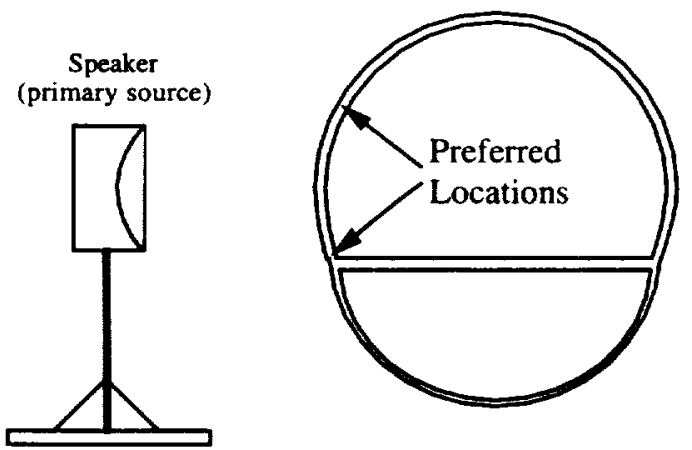

Figure 8. Diagram of Test Setup

Figure 7 shows the locations of the best 8 and worst 8 actuators based on their individual noise reduction capability and for both the constrained and unconstrained cases. The side of the aircraft facing the speaker (primary source) is favored in both cases. It can also be seen that the center of the section around the windows is a preferred location. This area is directly in line with the axis of the primary source (see figure 8). The worst areas for actuator placement appear to be on the far side of the aircraft and along the edges of the section. These characteristics seem to hold regardless

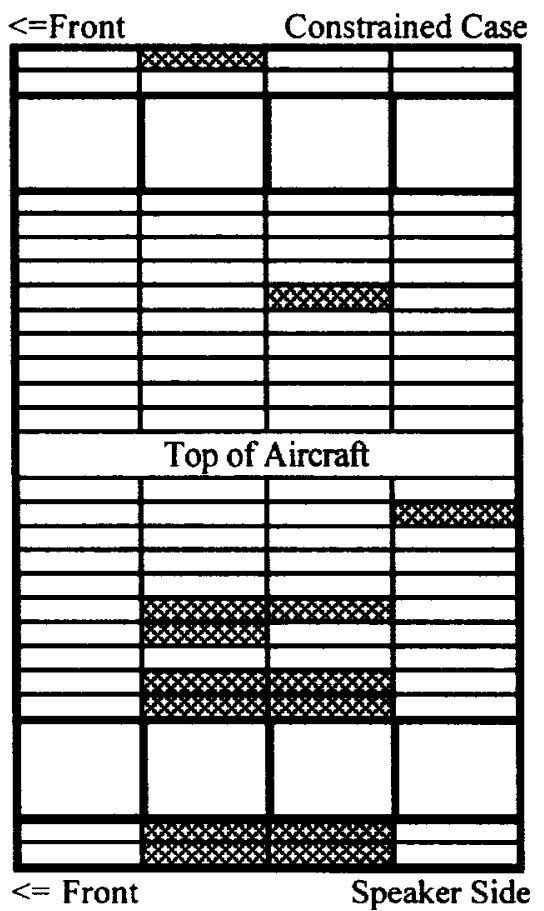

of constraint level and seem to be indicative of a general trend for this configuration.

\section{Results}

The optimized location of 14 actuators selected by tabu search are shown for both the constrained and unconstrained cases in figure 9. The constrained set of actuators produced a $4.6 \mathrm{~dB}$ reduction in noise level using at most $2.25 \mathrm{~V}$ rms of force while the unconstrained set of actuators produced $17.8 \mathrm{~dB}$ reduction. Notice that, unlike the previous single actuator example, the actuator array selected in the constrained case is quite different from that selected in the unconstrained case. The dominance of the speaker side of the aircraft is not apparent for the unconstrained case. As will be shown in the following, the unconstrained optimization can effectively group weaker actuators to achieve greater noise reduction at the expense of much greater forces.

The tabu search convergence history helps explain the difference between constrained and unconstrained optimizations. Figure 10 compares the cost function, eq. (4), for the two cases (uniform-constrained and unconstrained) as a function of tabu search iteration number. (Note that only improved states are shown on these convergence history plots.) Both cases are initialized with the same set of actuators. The unconstrained case evaluates this initial set and returns a $10 \mathrm{~dB}$ noise reduction potential using $128 \mathrm{Vrms}$ of

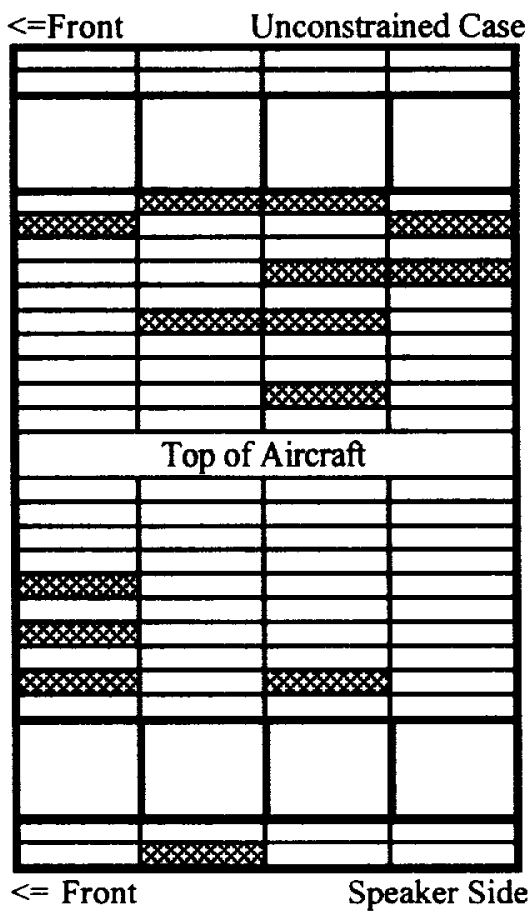

Figure 9. Constrained and Unconstrained Actuator Sets 


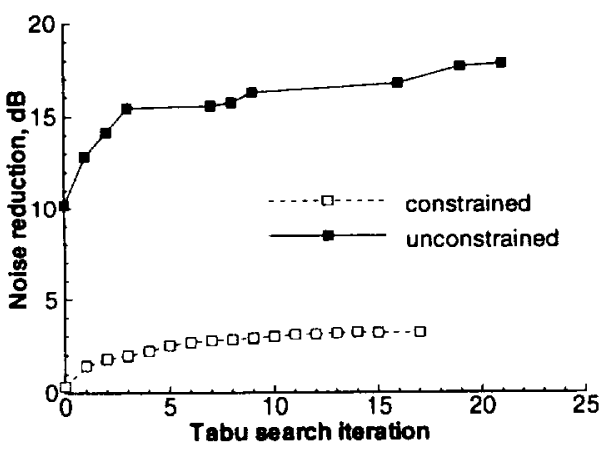

Figure 10. Cost Function History

force. The constrained case is able to achieve only 0.3 $\mathrm{dB}$ of noise reduction due to the high penalty that the control effort incurs. In both cases tabu search is able to increase noise reduction by selecting new sets of actuators. However, in the unconstrained case, actuators can be selected solely on their ability to reduce noise while in the constrained case actuators must reduce noise and control effort. It is not surprising that two different sets of actuators are identified.

The two types of constraining methods, uniform and CONMIN, are compared in an optimization of actuator subsets of size 14. The uniform constraint method is fast and efficient ( 3 minutes processing time) but returns sub-optimal noise reduction estimates. The CONMIN method returns optimal noise reduction estimates but requires much more computation ( $20 \mathrm{hrs}$. processing time). The final actuator sets returned by both techniques differ by only one actuator. When the noise reduction capability of both sets is computed using CONMIN, they differ by only a few tenths of a $\mathrm{dB}$ (uniform: $4.5 \mathrm{~dB}$ vs. CONMIN $4.7 \mathrm{~dB}$ ).

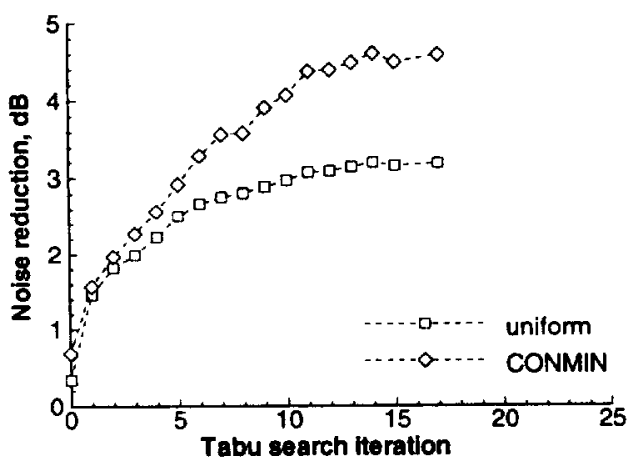

Figure 11. Trace of Uniform Search Re-evaluated Using CONMIN

This raises the question as to whether the more accurate CONMIN procedure finds better actuator sets. Figure 11 is a plot of the uniform-constrained cost function of fig. 10 compared to the same trace history re-evaluated using CONMIN. The plot illustrates the capability of the uniform method to correctly predict noise reduction trends even though it underestimates the actual value. These results indicate that the uniform constraint method is an effective cost function when used with tabu search.

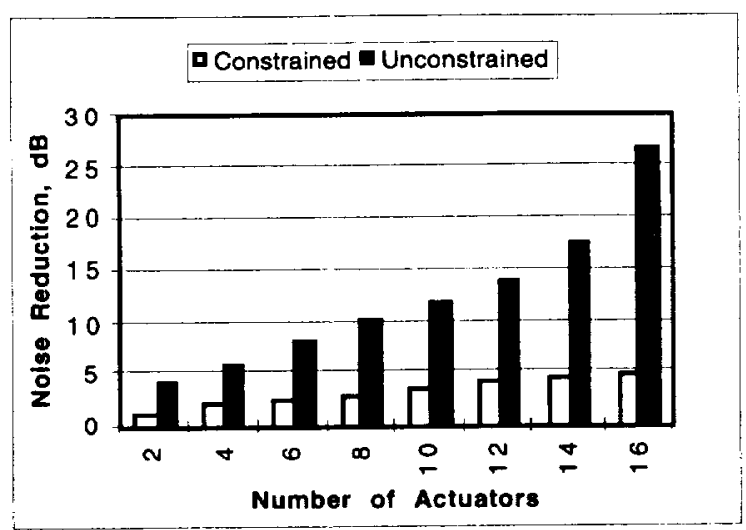

Figure 12. Noise Reduction vs. Number of Actuators

As mentioned earlier, the optimization procedure chooses an optimum actuator set for a fixed number of actuators. Insight into what might be an optimum number of actuators can be gained from plots of noise reduction and actuator force versus number of actuators. Figure 12 is a graph of noise reduction as a function of number of actuators for actuator sets optimized under constrained and unconstrained conditions. As expected, the amount of noise reduction increases with increasing actuators. However, the constrained case seems to approach a limit of $5 \mathrm{~dB}$ while the unconstrained case continues to increase. This is expected due to the inexhaustible availability of force for the unconstrained case.

Figure 13 is a graph of maximum control force as a function of number of actuators, i.e., at least one actuator in the set achieved the force plotted. However, all the forces are unconstrained, meaning that the

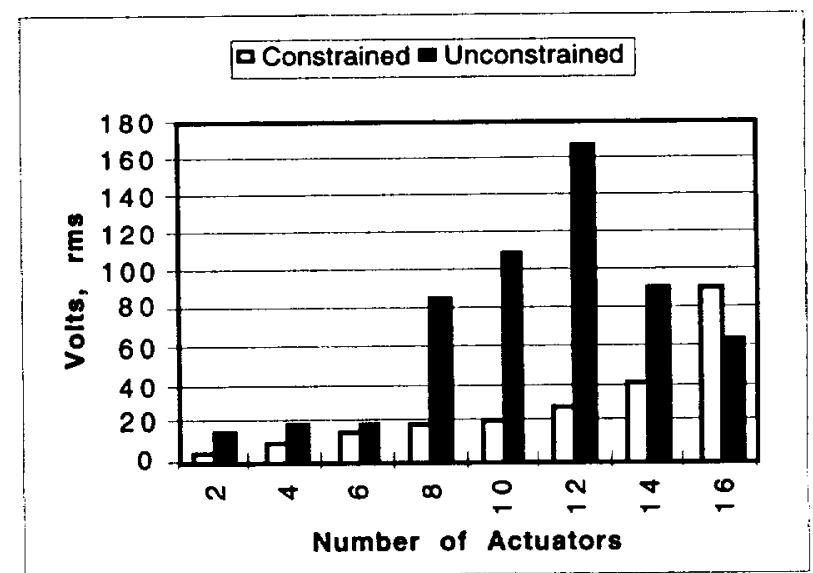

Figure 13. Maximum Actuator Force 
actuator subsets optimized under constrained conditions were reevaluated using the unconstrained solution, eq. (3). Notice that a point is reached for both cases where the force increases dramatically. Detailed analysis has shown that, as the actuator subset size increases, the optimization procedure begins selecting poorer performing actuators. For the unconstrained case this occurs at 8 actuators when the worst actuator with the lowest coherence is selected. The constrained case never selects the worst actuator, but, it is apparent that there are not 16 good actuator locations available. The point of diminishing returns is reached at 14 actuators.

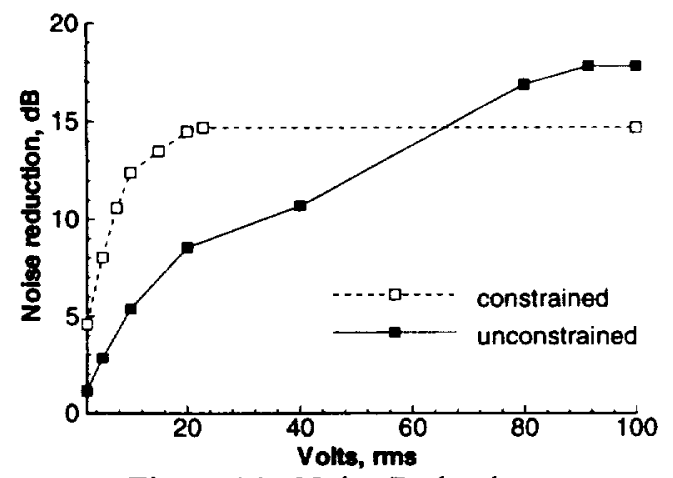

Figure 14. Noise Reduction vs. Force Constraint

The ability for a particular actuator set to perform at force levels other than the constraint level for which it was optimized is an important consideration. Figure 14 plots the noise reduction for 2 actuators sets (one optimized with constraint and one without) over the range of force from $2.25 \mathrm{Vrms}$ to $100 \mathrm{Vmms}$. It can be seen that the constrained actuator set has a greater potential for reducing noise at realistic levels, i.e., $<<$ $20 \mathrm{Vrms}$, than the unconstrained set.

\section{Conclusions}

Actuator performance, i.e., the noise reduction achieved per unit application of force, has been shown to be strongly dependent on actuator location. In general, actuators placed on the same side of the aircraft as the primary source have better performance than actuators on the opposite side. The best performing locations are clustered around areas of the fuselage normal to the plane wave propagating from the primary source.

Actuator performance varies with applied force. Some actuator locations deliver good performance at low force levels and average performance at high forces. Other actuators with poor performance at low levels have average performance at high forces. For the purposes of selecting an optimized actuator set, it is important to apply a reasonable force constraint. Precise duplication of the target system's force (power) specification is not necessary. Constrained actuator sets perform well over a broad range of forces. It has been shown that optimized, but unconstrained, actuator sets can have as members poorly performing actuators which require large amounts of force. This underscores the need to constrain the forces used during optimization.

The uniform constraint method is an efficient way of applying force constraint in the tabu search cost function for multi-frequency optimization. Although not an accurate predictor of noise reduction, the results indicate that the uniform method captures the relative ranking of the actuator sets in tabu search neighborhoods, thus enabling optimization.

\section{References}

1. Palumbo, D.L.; Padula, S.L.; Lyle, K.H.; Cline, J.H.; and Cabell, R.H.: "Performance of optimized Actuator and Sensor Arrays in an Active Noise Control System", NASA TM 110281 Sept. 1996.

2. Padula, S. L.; and Kincaid, R. K.: "Aerospace Applications of Integer and Combinatorial Optimization", NASA TM 110210, October 1995.

3. Rossetti, D.J.; Jolly, M.R., and Southward, S.C.: "Control Effort Weighting in FeedForward Adaptive Control System", J. Acoust. Soc. Am., Vol 99, No. 5, 1996, pp 2955-2964.

4. Mathur, G.; Tran, B.; and Simpson, M.: "Broadband Active Structural Acoustic Control of Aircraft Cabin Noise - Lab Tests", Proceedings $3^{\text {rd }}$ AIAA/CEAS Aeroacoustics Conference, AIAA-971636, May, 1997.

5. Elliot, S.J.; Stothers, I.M.; and Nelson, P.A.: "A Multiple Error LMS Algorithm and its Application to the Active Control of Sound and Vibration", IEEE Transactions on Acoustics, Speech and Signal Processing, pp. 1423-1434, 1987.

6. Nelson, P.A. and Elliott, S.J., "Active Control of Sound", Academic Press Ltd., 1992, pp 416-419.

7. Lester, H.C. and Silcox, R.J.: "Active Control of Interior Noise in a Large Scale Cylinder Using Piezoelectric Actuators", NASA/SAE/DLR 4th Aircraft Interior Noise Workshop, May 19, 1992.

8. Elliot, S.J.; Boucher, C.C.; and Nelson, P.A.: "The Behavior of a Multiple Channel Active Control System", IEEE Trans. Signal Process, Vol. 40, No. 5, 1992.

9. Vanderplaats, Garret N: "CONMIN - A FORTRAN Program for Constrained Function Minimization User's Manual", NASA TMX-62,282, August 1973. 